\title{
TRADUZIONE, ADATTAMENTO E VALIDAZIONE DI UN QUESTIONARIO SU MOTIVAZIONE E DISPOSIZIONE VERSO LA SCRITTURA E SUO IMPIEGO NELLO STUDIO DELL'AUTOPERCEZIONE DI CLASSI INTERE E STUDENTI MULTILINGUI
}

Letizia Lazzaretti

\section{INTRODUZIONE}

Scrivere non è compito facile da nessun punto di vista. Per organizzare un buon testo occorre infatti prestare attenzione a numerosi fattori, di natura sia cognitiva (cfr. Galbraith, 2009; MacArthur, Graham, 2016; Torrance, Galbraith, 2006) che socioculturale (cfr. Bazerman, 2011; 2016; Prior, 2006): la struttura logica del brano, la comprensibilità delle informazioni, l'adeguatezza al destinatario e alla cultura di riferimento e molto altro.

Tuttavia, i problemi che talvolta affliggono lo studente e gli impediscono di maturare un buon rapporto con la scrittura non riguardano solamente le competenze linguistiche e testuali, ma hanno a che fare anche con fattori di natura emotiva, affettiva e motivazionale (cfr. Boscolo, Hidi, 2007; Hidi, Boscolo, 2006). Per fare alcuni esempi, la scrittura è ancora oggi in molti contesti trattata come un'attività solitaria e riflessiva, contornata da pochi stimoli esterni e occasioni di collaborazione. Il ragionamento rispetto al suo funzionamento è scarso, i supporti e le strategie forniti sono limitati, i feedback giungono a distanza di tempo, quando ormai lo studente ha riservato la sua attenzione ad altro e ha dimenticato le difficoltà emerse durante quello specifico compito. I riferimenti al mondo esterno e agli scopi quotidiani sono spesso assenti, aspetto che non permette allo studente di assimilare il valore e l'utilità della scrittura rispetto ai propri scopi quotidiani e futuri (Graham, 2019). Parlando più nello specifico dei costrutti motivazionali associati a questa pratica, un aspetto di grande influenza sul processo di scrittura sono le credenze maturate a proposito di sé come scrittore e sullo scrivere in generale, potenti precursori dell'atteggiamento con cui poi ci si approccerà al compito stesso (White, Bruning, 2005): a tal proposito, la scelta di metodi, strategie, obiettivi e impegno da dedicare allo scritto sarà estremamente influenzata dalle convinzioni che gli studenti hanno maturato sul processo di scrittura stesso (McCarthy, Schmeck, 1988; Van Rossum, Schenk, 1984).

Altro fattore in grado di influenzare sia l'apprendimento in generale che la scrittura nello specifico è l'interesse (Boscolo, 2012), definito come quel peculiare tipo di relazione che si instaura tra un individuo e un oggetto o situazione all'interno di un determinato contesto (Hidi, Baird, 1986; Renninger et al., 1992). A tal proposito, è emerso che per mantenere un interesse stabile e duraturo verso la scrittura è necessario equipaggiare l'apprendente con strumenti concreti, strategie, metodi e conoscenze processuali, nonché trasmettere loro il valore di questa pratica, l'importanza di un lavoro strutturato e la

\footnotetext{
${ }^{1}$ Università degli Studi di Modena e Reggio Emilia.
} 
(C) Italiano LinguaDue 2. 2021. L. Lazzaretti, Tradurione, adattamento e validazione di un questionario su motivazione e disposizione verso la scrittura e suo impiego nello studio dell'autopercezione di classi intere e studenti multilingui

consapevolezza delle proprie possibilità di riuscita (Boscolo, 2012; Sansone, Thoman, 2005).

Da questo discorso emergono i due ulteriori macro costrutti associati alla scrittura: autoregolazione e autoefficacia. La prima è intesa come la capacità di regolare autonomamente i propri compiti e apprendimenti, gestendo consapevolmente tempi, risorse, obiettivi e strategie (cfr. Santangelo et al., 2016; Zimmerman, 2002; Zimmerman, Kitsantas, 1997); la seconda riguarda la valutazione che ogni essere umano compie su di sé e sulle sue presunte possibilità di riuscire in un determinato compito (Bandura, 1986; Bruning, Kauffman, 2016). Di conseguenza, per scrivere bene occorre sia imparare a gestire questo compito complesso in tutte le sue sfaccettature, sia acquisire una certa consapevolezza circa le proprie conoscenze e abilità in relazione ad esso.

Per tali ragioni, e data l'importanza di valutare non solo i fattori legati alla qualità del testo, ma anche le percezioni relative al valore, alle competenze e agli sforzi associati alla pratica di scrittura, si propone qui la traduzione italiana di un questionario in lingua inglese utile per ricavare informazioni di questo genere dagli studenti. Data la minor disponibilità di strumenti in lingua italiana, per uso sia didattico che di ricerca, rispetto a quelli esistenti in inglese, si è scelto di lavorare su uno strumento che fosse breve e di facile utilizzo in entrambi i settori: un questionario di undici item relativo alla disposizione a scrivere, rivolto a studenti di scuola primaria e secondaria di primo grado. A parere degli autori dello strumento (Piazza, Siebert, 2008), tale atteggiamento deriva proprio dall'intreccio di tutti i costrutti motivazionali precedentemente passati in rassegna, dunque si tratta di un concetto molto ampio e sfaccettato. Il secondo capitolo di questo contributo sarà dedicato al processo di costruzione dello strumento: traduzione, adattamento e successiva validazione, suddivisa in una fase pre-pilota e un pilotaggio vero e proprio.

Le prestazioni del questionario sono state poi osservate in una ricerca di tipo quasisperimentale (cfr. Benvenuto, 2015) relativa a percezioni di scrittura e qualità del testo, di cui si tratterà nel terzo capitolo. In particolare, sono stati confrontati due gruppi di studenti di quinta primaria e terza secondaria di primo grado: il primo, sperimentale, composto da ragazzi che hanno seguito un progetto di educazione linguistica di stampo attivo, collaborativo e inclusivo, contemporaneamente attento agli aspetti cognitivi, sociali e motivazionali che si celano dietro il processo di scrittura; il secondo, di controllo, composto da alunni che hanno seguito la normale programmazione didattica. Per lo studio si è fatto uso del questionario e di alcune misure olistiche per valutare la qualità di produzioni scritte, al fine di indagare gli effetti, su motivazione e abilità di scrittura, apportati dal Progetto su coloro che vi hanno preso parte. Il confronto è avvenuto non solo per gruppi interi, ma anche isolando e confrontando i due sottogruppi di multilingui, intesi come parlanti attivi di una o più lingue oltre all'italiano e alle lingue apprese a scuola.

Il quarto ed ultimo capitolo riporterà invece le conclusioni del presente lavoro, riflettendo sia sullo strumento realizzato che sulla ricerca effettuata.

\section{TRADUZIONE E VALIDAZIONE DEL QUESTIONARIO}

Come anticipato, nel panorama italiano vi è maggior carenza, rispetto ai contesti anglofoni, di strumenti specifici per lo studio delle percezioni di scrittura, nonostante sia nota l'importanza di affiancare allo studio delle produzioni scritte anche un monitoraggio dei fattori affettivi e motivazionali legati a questo complesso processo. Per tale ragione riportiamo qui il processo di costruzione della versione italiana di un questionario su 
(C) Italiano LinguaDue 2. 2021. L. Lazzaretti, Tradurione, adattamento e validazione di un questionario su motivazione e disposizione verso la scrittura e suo impiego nello studio dell'autopercezione di classi intere e studenti multilingui

motivazione e disposizione verso la scrittura a scrivere rivolto a studenti di scuola primaria e secondaria di primo grado.

\subsection{Lo strumento: tradurione, adattamento e opzioni di risposta}

Lo strumento scelto per la traduzione è il Writing dispositions scale for elementary and middle school students (Piazza, Siebert, 2008), che indaga la motivazione e, letteralmente, la disposizione (disposition) degli studenti verso la scrittura. Tale costrutto è inteso dagli autori come una caratteristica personale che riguarda il temperamento e il carattere dell'apprendente, più propriamente

un ampio costrutto facente parte del dominio affettivo tramite cui gli scrittori apportano alla loro scrittura risorse come l'autodisciplina, la perseveranza di fronte alle difficoltà, la tolleranza dell'ambiguità, l'autonomia, la disponibilità a correre rischi, la motivazione, l'autoefficacia e l'interesse (Piazza, Siebert, 2008: 275 , auth. trans).

La disposizione verso la scrittura è il risultato dell'interazione reciproca tra diversi fattori di natura sociale (importanza del compito, obiettivi di riuscita), cognitiva (abilità preesistenti, conoscenze a proposito del compito) e strategica (strategie di scrittura, padronanza dei processi). Dall'intreccio di tali fattori emergono appunto quelle manifestazioni affettive e comportamentali definibili come disposizione verso la scrittura. In particolare, per indagare questo costrutto dall'ampia portata, i ricercatori hanno costruito un questionario da undici item, strutturato nelle seguenti sottoscale:

- fiducia (confidence);

- persistenza (persistence);

- passione (passion).

Per fiducia si intende, appunto, il credere fortemente nelle proprie capacità di scrittura e, di conseguenza, la sicurezza circa la propria autoefficacia nei confronti di questo compito. Tale credenza si collega al concetto di teoria incrementale dell'intelligenza (cfr. Dweck 2000; Dweck, Grant, 2008): aver fiducia in sé e in ciò che si può fare con le proprie capacità prevede anche che si sia sicuri di poter migliorare, in altre parole che la buona scrittura non sia un dono di natura conferito a pochi, ma un processo complesso che si può via via imparare a padroneggiare.

La persistenza ha invece a che fare con la disponibilità dell'individuo a dedicarsi alla scrittura e la volontà di protrarre questo impegno in modo prolungato nel tempo, senza arrendersi alla prima difficoltà o al primo segno di stanchezza. Chi è più dedito ad impiegare energie nello scrivere è anche più propenso a pianificare in modo chiaro e dettagliato il proprio discorso prima di scriverlo e a revisionarlo durante o dopo la stesura; in altre parole è maggiormente disposto a seguire gli step di scrittura e a ritornare più e più volte sul suo lavoro al fine di perfezionarlo.

Infine, la passione ha a che fare con il desiderio, anche protratto nel tempo, di dedicarsi a compiti di scrittura e con la piacevolezza e il godimento che si trae dal cimentarsi in essa. Si tratta insomma del piacere di scrivere provato dagli individui, del loro essere ben disposti a scrivere, trovandosi non di rado a farlo anche per loro volontà e diletto personale, senza essere in qualche modo "obbligati" da scuola e insegnante. Pare inoltre che coloro a cui piace la scrittura siano anche più inclini a coglierne il valore e l'importanza 
(C) Italiano LinguaDue 2. 2021. L. Lazzaretti, Tradurione, adattamento e validazione di un questionario su motivazione e disposizione verso la scrittura e suo impiego nello studio dell'autopercezione di classi intere e studenti multilingui

per la loro vita attuale e futura, insomma che si impegnino in questa attività non solo perché a loro piace o perché fa parte di ciò che viene insegnato a scuola, ma anche perché si rendono conto di quanto sia importante saperla padroneggiare con un minimo di dimestichezza.

Come anticipato, il primo passo per la realizzazione dello strumento è stato quello della traduzione. Questa prima fase di lavoro si è svolta in due momenti: traduzione letterale e, laddove necessario, aggiustamenti di significato o adattamenti al contesto scolastico italiano. Per spiegarsi meglio, si propongono di seguito due esempi:

Item originale: I take time to solve problems in my writing.

Traduzione letterale: Mi prendo del tempo per risolvere i problemi del mio testo scritto.

Traduzione con aggiustamento di significato: Cerco di sistemare $i$ miei testi al meglio, anche se ci vuole un po' di tempo.

In questo caso la traduzione fedele all'originale è parsa complessa da decifrare per un ipotetico destinatario dello strumento, motivo per cui si è optato per una riformulazione più chiara, seppur dotata di una sfumatura di significato lievemente diversa dall'originale.

Item originale: Writing is my favorite subject in school.

Traduzione letterale: Scrivere è la mia materia scolastica preferita.

Traduzione con adattamento al contesto: Scrivere è la mia attività scolastica preferita.

In questo secondo caso si è sostituito il termine materia (subject) con il termine attività. Nella scuola italiana, infatti, la scrittura non è una disciplina vera e propria, bensì un'attività comune a diversi momenti della routine scolastica. Per rendere il quesito maggiormente vicino all'esperienza quotidiana degli studenti italiani, si è optato quindi per questa modifica.

Una volta ottenuta la prima versione tradotta dello strumento, si è passati alla scelta della scala di risposta. Il questionario di Piazza e Siebert è costruito su una scala Likert a cinque livelli (1-fortemente d'accordo; 5-fortemente in disaccordo), ma nel nostro caso si è preferito optare per linee continue da 0 a 100 della lunghezza di 10 centimetri. A seguire un esempio (Figura 1).

Figura 1. Scala continua di risposta

per niente poco così così molto moltissimo

Agli alunni è stato spiegato che potevano rispondere ai quesiti tramite una crocetta posta ovunque sulla linea: sopra alle parole, tra di esse o addirittura in corrispondenza dei due estremi. In tal modo ogni risposta, misurata poi con un righello, avrebbe potuto posizionarsi su un qualsiasi valore intero compreso nella scala $0-100$. Scegliendo una scala di questo tipo è possibile trattare i numeri sulla linea come effettive cifre e come unità continue, potendo dunque effettuare, in maniera metodologicamente corretta, operazioni con essi: somme, divisioni, medie e quant'altro (Chyung et al., 2018). Tutto questo risulterebbe un po' meno adeguato con una scala Likert, i cui valori sono ordinali, in altre parole servono a ordinare su ranghi determinate qualità (ad es. 1 corrisponde a per niente, 2 a poco, 3 ad abbastanz̧a, 4 a molto e così via), ma non rappresentano veri e propri valori numerici sui quali intervenire con operazioni. Sulla linea di risposta si è poi scelto di 
(C) Italiano LinguaDue 2. 2021. L. Lazzaretti, Tradurione, adattamento e validazione di un questionario su motivazione e disposizione verso la scrittura e suo impiego nello studio dell'autopercezione di classi intere e studenti multilingui

inserire anche alcune parole di riferimento, che potessero aiutare gli studenti a comprendere il senso e il verso dello strumento (come nell'esempio in Figura 1), pur rendendo chiara ed esplicita agli alunni la totale libertà nell'apporre le loro risposte dove meglio ritenuto. Come spiega poi Bailey (1978/1995: 65), la scelta di questo genere di scala è riconducibile a motivazioni non solo di natura statistica e matematica, ma anche di natura emotiva:

se l'intervistato pensa che [quelle a lui richieste] siano informazioni delicate [o complicate], avrà minori difficoltà ad indicare [un parere] approssimativo all'interno di un determinato intervallo.

In altre parole, se gli studenti non hanno un'idea del tutto precisa e univoca su un certo argomento o, diversamente, non si sentono completamente sicuri nel collocarsi in una precisa posizione (ad esempio sopra una delle parole guida), con questa linea di risposta hanno modo di esprimere anche un parere approssimativo, a metà tra due posizioni, rendendo ancora più veritiero e affidabile il loro pensiero.

Il questionario tradotto presentava lo stesso numero di item di quello originario e si articolava su linee continue di risposta comprendenti due versioni differenti di parole guida, a seconda del senso dei quesiti: per niente, poco, cosi così, molto, moltissimo oppure mai, raramente, qualche volta, spesso, sempre.

Da questo momento in poi ha preso avvio la fase di validazione dello strumento, che si è svolta in due momenti: una sessione pre-pilota e uno studio pilota.

\subsection{Fase pre-pilota: comprensibilità}

La sessione pre-pilota, finalizzata a raccogliere informazioni circa la comprensibilità di ogni item e della scala di risposta, ha coinvolto una sola classe, una quarta primaria (24 studenti) appartenente ad un Istituto Comprensivo situato in una provincia del nord Italia. Si è scelto di utilizzare una sola classe in quanto, a parere di alcuni (ad es. Alderson et al., 1995; Caselli, 2007), non è necessario che il pretesting coinvolga un ampio campione: è sufficiente un gruppo ristretto che in qualche modo riproduca le caratteristiche degli individui a cui si vorrà somministrare quello strumento. In tal senso la classe scelta riproduceva le caratteristiche dei campioni di nostro interesse, già brevemente menzionati nell'introduzione e su cui si tornerà in seguito: un gruppo variegato di studenti italiani, diversi dei quali di origine straniera, con alcuni giunti in Italia da non troppi anni.

Lo studio della comprensibilità di questo strumento è avvenuto in concomitanza al pre-pilotaggio di altri tre questionari, dei quali non si farà menzione in questo contributo. La somministrazione si è svolta in due giornate, la prima delle quali si è così articolata:

1. Prima fase: si è prodotto un numero di fascicoli pari al totale degli studenti della classe. In ogni fascicolo era presente la copia di uno solo dei quattro strumenti, per un totale di sei copie di ogni questionario. Le copie sono state distribuite in quattro pile distinte, una per tipo di questionario, rivolte a faccia in giù per non mostrarne il contenuto;

2. Seconda fase: ogni alunno, chiamato singolarmente fuori dalla classe ${ }^{2}$, ha scelto in modo del tutto casuale un questionario da una delle quattro pile. Una volta girato il plico, ha letto le istruzioni e compilato lo strumento attraverso una discussione

2 Si è proceduto in questo modo, come suggerito da Phillips e Clancy (1972), per far lavorare gli studenti in tutta tranquillità ed evitare il più possibile distorsioni dovute a disagio e desiderabilità sociale. 
(C) Italiano LinguaDue 2. 2021. L. Lazzaretti, Tradurione, adattamento e validazione di un questionario su motivazione e disposizione verso la scrittura e suo impiego nello studio dell'autopercezione di classi intere e studenti multilingui

guidata, comprendente vere e proprie domande dirette ed il metodo indiretto del pensiero ad alta voce (Lumbelli, 2009). Per prima cosa si è chiesto di leggere la pagina di istruzioni e di commentarla, in modo tale da rendersi conto dell'effettiva efficacia della stessa. Una volta sondata la comprensione delle regole di compilazione, si è passati al questionario vero e proprio, talvolta ponendo domande dirette, talvolta ascoltando le considerazioni dei bambini. Per rendere questa "intervista" il più possibile standardizzata sono state prodotte delle definizioni dei termini ritenuti più difficili e del senso che si intendeva attribuire ad ogni item, in modo tale da fornire sempre la stessa risposta ad eventuali domande di chiarimento (Presser, Zhao, 1992). Inoltre, al fine di non accontentarsi di una conferma di superficie, di fronte a commenti poco chiari e questioni più complesse si è dato modo agli alunni di motivare ulteriormente le proprie scelte o considerazioni, al fine di accertarsi effettivamente dell'avvenuta comprensione del quesito o dell'istruzione in questione (Lazarsfeld, 1944).

A seguito di questa prima somministrazione sono state individuate tutte le difficoltà di comprensione generate dallo strumento. I principali problemi sono stati due:

- formulazione linguistica di alcuni item: il lessico e la struttura di alcuni quesiti risultavano di difficile comprensione, motivo per cui certe frasi sono state semplificate. Ad esempio, l'item Cerco di pensare a diversi modi di scrivere le cose anche se questo mi richiede un po' di tempo è stato riformulato come Anche se ci vuole un po' di tempo, cerco di pensare a diversi modi di dire la stessa cosa, per capire qual è il migliore;

- doppia negazione nell'incontro tra testo dell'item e opzioni di risposta. Poniamo un esempio: di fronte all'item Non sono tanto bravo a scrivere è capitato che diversi alunni decidessero di porre la crocetta in prossimità di per niente, volendo però intendere che loro sono "per niente bravi a scrivere", non, come il senso del quesito richiederebbe, che loro non si sentono per niente "non bravi a scrivere", dunque si reputano bravi.

Nel primo caso, come si diceva, sono stati riformulati i quesiti dove necessario, mentre nel secondo caso si è scelto di modificare, per tutti gli item, le parole guida poste sotto alla linea di risposta, scegliendo una forma unica per tutti i quesiti. Ci si è, a questo punto, trovati indecisi su due possibili alternative: la prima assolutamente falso, abbastanza falso, né vero né falso, abbastanza vero, assolutamente vero, la seconda non è per niente vero, non è molto vero, non è né vero né falso, è abbastanza vero, è proprio vero. Questa questione è stata oggetto di verifica della seconda giornata di somministrazione, che si è così articolata:

1. Prima fase. Sono stati nuovamente realizzati ventiquattro fascicoli da un solo questionario, uno per ogni alunno della classe: dei sei questionari per ogni tipo, tre copie contenevano la prima versione delle opzioni di risposta, le altre tre contenevano la seconda versione.

2. ogni studente ha scelto casualmente un questionario (avendo cura di escludere dalla scelta, di volta in volta, lo strumento già compilato nella precedente sessione) e lo ha letto e completato in maniera dialogata, al fine di valutare i progressi in termini di comprensione e agevolezza nella compilazione.

Da questa seconda sessione è parso che i quesiti riformulati fossero di più facile comprensione per gli studenti. Inoltre, la prima opzione di risposta (assolutamente falso, abbastanza falso, né vero né falso, abbastanza vero, assolutamente vero) è sembrata più adatta a risolvere il problema della doppia negazione e più semplice da interpretare, motivo per cui è stata scelta per la versione definitiva dello strumento. 
(C) Italiano LinguaDue 2. 2021. L. Lazzaretti, Tradurione, adattamento e validazione di un questionario su motivazione e disposizione verso la scrittura e suo impiego nello studio dell'autopercezione di classi intere e studenti multilingui

\subsection{Fase pilota: affidabilità interna}

A seguito di tutte le modifiche apportate è stato realizzato lo strumento definitivo, poi denominato Questionario su quanto ti piace scrivere. Il questionario così composto è stato oggetto di una seconda fase di validazione, incentrata sull'affidabilità interna. Il campione questa volta era composto da nove classi di un Istituto Comprensivo situato in una provincia del nord Italia: tre quarte primarie, due quinte primarie, una prima secondaria di primo grado, due seconde secondarie di primo grado e una terza secondaria di primo grado, per un totale di 179 studenti.

Seguendo l'esempio di Piazza e Siebert, è stata calcolata la consistenza interna sia delle tre sottoscale separate, sia dell'intero strumento. A tale scopo si è scelto di utilizzare l'indice Omega (McDonald, 1970; 1999), un'alternativa meno conosciuta ma metodologicamente più corretta rispetto al tradizionale Alpha di Cronbach (Dunn, et al., 2014).

Tabella 1. Indice Omega per sottoscale e intero questionario

\begin{tabular}{|c|c|}
\hline Sottoscala Fiducia & $\begin{array}{c}\text { Omega: } 0.75 \\
95 \% \text { CI: low } 0.68 ; \text { up } 0.82\end{array}$ \\
\hline Sottoscala Persistenza & $\begin{array}{c}\text { Omega: } 0.61 \\
95 \% \text { CI: low } 0.52 ; \text { up } 0.71\end{array}$ \\
\hline Sottoscala Passione & $\begin{array}{c}\text { Omega: } 0.89 \\
95 \% \text { CI: low 0.86; up } 0.91\end{array}$ \\
\hline Intero questionario & $\begin{array}{c}\text { Omega: } 0.89 \\
95 \% \text { CI: low 0.86; up } 0.91\end{array}$ \\
\hline
\end{tabular}

La Tabella 1 riporta i valori puntuali di Omega e i relativi intervalli di confidenza al 95\%, che indicano, appunto, l'intervallo di valori entro cui potrebbe cadere la cifra in questione nel 95\% delle future osservazioni con ulteriori campionamenti casuali di popolazione. Dall'osservazione dei valori in tabella, e tenendo a mente l'eterogeneità dei pareri su quali siano gli intervalli numerici indicativi della buona consistenza di uno strumento $^{3}$, possiamo considerare buona la situazione della sottoscala Fiducia, sufficiente quella della sottoscala Persistenza e ottima quella della sottoscala Passione e dell'intero strumento.

Per ciò che riguarda la scala sulla Persistenza, essa mostra i valori più bassi sia qui che nello studio originario di Piazza e Siebert (2008: 279), lasciando intendere che la sua consistenza interna sia effettivamente inferiore a quella delle altre scale. Ciò detto, data l'eterogeneità dei pareri in letteratura e considerando che si tratta del primo sondaggio di

\footnotetext{
${ }^{3}$ Secondo alcuni pareri, ad esempio quello di Cortina (1993), un valore da 0.70 in poi è da considerarsi adeguato, ancor meglio da 0.80 a crescere; per altri, come Cicchetti e Sparrow (1990), i valori da 0.70 a decrescere sono addirittura significativi di assoluta inadeguatezza, mentre quelli da 0.90 in poi indicano una scala eccellente. Di opinione diversa sono coloro che, come Green et al. (1977) e Vaske et al. (2017), ritengono, per ricerche di natura umanistica, spesso incentrate su costrutti d'ampio raggio e difficilmente incanalabili in scale del tutto unidimensionali, sufficientemente adeguati valori da 0.60-0.65 a salire. C'è anche chi, ad esempio Streiner (2003), condanna coefficienti troppo altri, superiori a 0.90, in quanto talvolta indicativi di item tutti troppo simili tra loro e, dunque, di una scala eccessivamente ridondante nei suoi contenuti.
} 
(C) Italiano LinguaDue 2. 2021. L. Lazzaretti, Tradurione, adattamento e validazione di un questionario su motivazione e disposizione verso la scrittura e suo impiego nello studio dell'autopercezione di classi intere e studenti multilingui

affidabilità su questo strumento, si è deciso di considerare tutti i valori emersi pienamente sufficienti per i nostri scopi, nonostante la condizione di minor consistenza della sopracitata scala sulla Persistenza. Lo strumento potrà poi essere suscettibile di modifiche e perfezionamenti in futuro, contemplando successive indagini di affidabilità.

Il questionario definitivo ottenuto a questo punto del lavoro è riportato in Appendice A.

\section{PRIMO IMPIEGO DELLO STRUMENTO: STUDIO DELL'AUTOPERCEZIONE DI SCRITTURA DI STUDENTI MONOLINGUI E MULTILINGUI}

A seguire sarà presentata e descritta la prima ricerca nella quale il Questionario su quanto ti piace scrivere è stato utilizzato. Lo scopo, come anticipato, era quello di ricavare informazioni su autopercezioni di scrittura e qualità delle produzioni scritte (testi narrativi) di studenti di quinta primaria e terza secondaria di primo grado, confrontando un gruppo sperimentale, che ha seguito per diversi anni un progetto di educazione linguistica, con un gruppo di controllo, che ha seguito la normale programmazione.

\subsection{Contesto di ricerca}

Lo studio qui presentato si inserisce nel più ampio quadro di ricerca del Progetto di formazione e ricerca-azione Osservare l'interlingua ${ }^{4}$, nato nel 2007 dalla collaborazione tra il Comune di Reggio Emilia e l'Università degli Studi di Modena e Reggio Emilia ${ }^{5}$. Il coordinamento e supervisione scientifica sono affidati al Prof. Gabriele Pallotti, mentre i destinatari sono gli studenti di scuola primaria e secondaria di primo grado. L'ambito d'intervento principale è l'educazione linguistica e riflessione sulla lingua in ambiente scolastico, con particolare attenzione verso la scrittura. Nei primi anni di sperimentazione la principale finalità del Progetto era quella di permettere l'integrazione, linguistica e affettiva, degli alunni con altra lingua madre, che in alcune delle scuole prese in esame dal gruppo di ricerca costituivano la stragrande maggioranza dell'utenza. In seguito i suoi obiettivi si sono ampliati, coinvolgendo anche gli studenti che hanno l'italiano come lingua madre, al fine di proporre ad intere classi, e non ai soli componenti ritenuti più in difficoltà, percorsi linguistici atti al miglioramento della produzione sia orale che scritta.

Come si diceva, il fine principale del Progetto è di natura linguistica: applicando il cosiddetto approccio dell'interlingua all'educazione linguistica (Selinker, 1972; Pallotti, 2017a), questo propone alle classi partecipanti percorsi educativi che coniugano una didattica attiva e per task (Ellis, 2003; Nunan, 2004) con un apprendimento il più possibile cooperativo e condiviso, lasciando costantemente spazio alla valutazione formativa (Black, William, 2009) e a sessioni di autovalutazione. La scrittura è affrontata nella sua dimensione cognitiva e processuale, in modo tale che gli alunni facciano il più possibile esperienza dei suoi diversi processi, sottoprocessi e strategie (Boscolo, 1990; 2002; White, Arndt, 1991). Le attività proposte, seguendo l'approccio sociocostruttivista (Vygotskij, 2006 [1926]; 1992 [1934]), privilegiano il formato di gruppo (piccolo o grande in base all'evenienza), in modo tale che tutti, coi propri pregi e limiti, siano partecipi dei lavori in

\footnotetext{
4 Per un approfondimento sulla metodologia didattica e di ricerca di Osservare l'interlingua si vedano: Borghetti et al. (2019), Ferrari, Burzoni (2018), Pallotti (2017b), Pallotti, Borghetti (2019), Pallotti et al. (2021), Pallotti, Ferrari (2019), Pallotti, Rosi (2017a; 2017b). Sito web: https://interlingua.comune.re.it/.

${ }^{5}$ La collaborazione con il Comune di Reggio Emilia è terminata nel 2019.
} 
corso e prestino il proprio contributo al raggiungimento dell'obiettivo finale. L'insegnante in tutto questo modifica il proprio intervento nella classe: da principale detentore del sapere e coordinatore delle attività a supporto e guida in caso di evenienza (King, 1993).

Le finalità del Progetto, tuttavia, non riguardano soltanto lo sviluppo di abilità linguistiche e l'apprendimento di strategie di comunicazione efficace, ma si rivolgono anche a obiettivi trasversali all'apprendimento. Tra questi spicca in particolare la volontà di costruire atteggiamenti positivi e motivati verso la buona ed efficace comunicazione verbale e la scrittura in particolare. In altre parole, si intende trasmettere un'idea di scrittura come qualcosa di effettivamente utile e arricchente, un mezzo per sviluppare consapevolezza linguistica e autonomia nella comunicazione, non solo una noiosa esercitazione scolastica. Oltre a ciò, come anticipato, il Progetto mira il più possibile all'integrazione di ogni componente della classe, in particolare di coloro che presentano origini geografiche e background linguistici differenti. I percorsi di volta in volta proposti, infatti, mirano a garantire un'educazione linguistica il più possibile inclusiva e democratica: tutti devono poter acquisire i mezzi necessari per comunicare e relazionarsi in modo efficace e per esercitare una presenza attiva e consapevole nella propria cultura di riferimento (Giscel, 1975).

Date queste premesse, è obiettivo di questo studio ricavare informazioni sull'efficacia a lungo termine del Progetto tramite dati sia di autopercezione che linguistici, per comprendere quali e quanti effetti, sia didattici che trasversali, esso ha prodotto negli studenti che vi hanno preso parte.

\subsection{Campione}

Il campione di questo studio è costituito da $226^{6}$ studenti suddivisi in tredici classi appartenenti a otto diversi Istituti Comprensivi situati in una provincia del Nord Italia. In particolare, nove di queste sono quinte primarie e quattro sono terze secondarie di primo grado. Tra queste classi, quelle sperimentali (cinque quinte primarie e due terze secondarie I grado) hanno partecipato al Progetto per un periodo variabile dai tre ai cinque anni, quelle di controllo (quattro quinte primarie e due terze secondarie I grado) hanno seguito la programmazione didattica prevista per l'anno scolastico di riferimento. Le informazioni sul campione sono riassunte in Tabella 2.

Tabella 2. Composizione gruppi

\begin{tabular}{|c|c|}
\hline Gruppo sperimentale & Gruppo di controllo \\
\hline 122 alunni & 104 alunni \\
\hline 74 femmine, 48 maschi & 61 femmine, 43 maschi \\
\hline 77 monolingui & 65 monolingui \\
\hline 45 multilingui, di cui 14 non nati in Italia & 39 multilingui, di cui 13 non nati in Italia \\
\hline
\end{tabular}

6 A seguito della raccolta dati sono stati esclusi gli studenti con certificazione DSA o L.104 e $\backslash$ o con conoscenza scarsa o quasi nulla della lingua italiana, dovuta ad arrivo molto recente in Italia o ad esigua esposizione alla lingua in ambiente extrascolastico. Si è proceduto in questo modo al fine di ottenere un campione le cui abilità di lettura, scrittura e comprensione non fossero eccessivamente ostacolate da difficoltà preesistenti, e che dunque gli studenti fossero in grado di svolgere al loro meglio le attività previste per la rilevazione (compilazione del questionario e scrittura di un testo). 
(C) Italiano LinguaDue 2. 2021. L. Lazzaretti, Tradurione, adattamento e validazione di un questionario su motivazione e disposizione verso la scrittura e suo impiego nello studio dell'autopercezione di classi intere e studenti multilingui

\begin{tabular}{|c|c|}
\hline Provenienza famiglie dei multilingui: & Provenienza famiglie dei multilingui: \\
6 Marocco; 3 Egitto; 1 Italia/S. Domingo; & 6 Marocco; 3 Moldavia; 3 Albania; \\
13 Albania; 4 Tunisia; & 7 Cina; 4 Ghana; 1 Senegal; 2 Nigeria; \\
1 Italia/Germania; 1 Sri Lanka; & 2 Ucraina; 1 Costa d'Avorio; 3 Tunisia; \\
2 Moldavia; 2 Pakistan; 4 Cina; & 1 Algeria; 2 Burkina Faso; 1 India; \\
1 Colombia; 1 Ungheria; 1 Ghana; & Italia/Marocco; 1 Ucraina; 1 Russia \\
$\begin{array}{c}\text { 1 Senegal; 1 Nigeria; 1 Italia/Brasile; } \\
\text { 1 Italia/Argentina; 1 Italia/Moldavia }\end{array}$ & \\
\hline $\begin{array}{c}\text { 102 entrati nei servizi educativi italiani con } \\
\text { la scuola dell'infanzia o prima }\end{array}$ & $\begin{array}{c}79 \text { entrati nei servizi educativi italiani con } \\
\text { la scuola dell'infanzia o prima }\end{array}$ \\
\hline $\begin{array}{c}\text { 11 entrati nei servizi educativi italiani al } \\
\text { primo anno di scuola primaria }\end{array}$ & $\begin{array}{c}\text { 15 entrati nei servizi educativi italiani al } \\
\text { primo anno di scuola primaria }\end{array}$ \\
\hline $\begin{array}{c}\text { entrati nei servizi educativi italiani tra i } \\
\text { sette e gli undici anni }\end{array}$ & $\begin{array}{c}10 \text { entrati nei servizi educativi italiani tra i } \\
\text { sette e gli undici anni }\end{array}$ \\
\hline
\end{tabular}

Come si può osservare dalle cifre in Tabella2, i gruppi sono piuttosto somiglianti, pur con qualche unità in più per quello sperimentale. In entrambi i raggruppamenti, poi, è presente una discreta quantità di multilingui, qui intesi come quegli alunni stranieri o di origine straniera che parlano attivamente una o più lingue oltre all'italiano, escluse le lingue apprese a scuola (cfr. Cook, 1999; Davies, 2004). Sono multilingui, ad esempio, gli alunni neo-arrivati in Italia (NAI) (cfr. Favaro, 2018), coloro che dalla nascita sono esposti contemporaneamente a due o più lingue, oppure gli beritage speakers, ossia quegli individui che alla nascita sviluppano solo la lingua parlata in famiglia, apprendendo poi nella primissima infanzia anche la lingua del luogo di residenza (cfr. Montrul, Polinsky, 2016). Per monolingui si intendono invece coloro che in famiglia hanno appreso solo la lingua italiana e conoscono altre lingue solo a livello scolastico. Sono monolingui anche gli studenti di origine straniera che non conoscono abbastanza della \e lingua \delle origini per poterne fare un uso effettivamente attivo e comunicativo.

\subsection{Obiettivi e domande di ricerca}

Il fine di questo studio è valutare gli effetti a lungo termine del progetto di educazione linguistica Osservare l'interlingua, che, oltre a insistere su processi e strategie efficaci di scrittura e comunicazione, annovera tra i suoi obiettivi trasversali la creazione di atteggiamenti positivi e motivati verso la scrittura e la riflessione sulla lingua. A tal proposito, le domande che hanno guidato questa indagine sono le seguenti:

1. la partecipazione alle attività del Progetto ha influito sulla qualità dei testi narrativi del gruppo sperimentale in termini di coesione, coerenza, completezza e comprensibilità del prodotto?

2. la partecipazione alle attività del Progetto ha influito sull'autopercezione degli studenti del gruppo sperimentale in termini di motivazione e disposizione verso la scrittura?

Oltre alle questioni motivazionali, il Progetto mira il più possibile all'inclusione di tutti gli studenti nelle attività comuni, in particolar modo i cosiddetti multilingui, in nome di un'educazione linguistica equa e democratica. Per questa ragione, si è scelto di confrontare classi sperimentali e di controllo non solo interamente, ma anche per soli multilingui, per 
(C) Italiano LinguaDue 2. 2021. L. Lazzaretti, Tradurione, adattamento e validazione di un questionario su motivazione e disposizione verso la scrittura e suo impiego nello studio dell'autopercezione di classi intere e studenti multilingui

comprendere se i risultati a gruppo intero si riflettessero o meno su quel gruppo di soggetti che, per ragioni linguistiche e socio-culturali, potrebbe presentare difficoltà maggiori. A tal proposito, l'ultima domanda che ha guidato questo lavoro è la seguente:

3. i risultati linguistici e di autopercezione raggiunti a gruppo intero si sono riflettuti anche sui soli alunni multilingui? I percorsi didattici proposti dal Progetto hanno contribuito ad accorciare le distanze tra i monolingui e gli studenti a vario titolo classificati come multilingui?

\subsection{Strumenti e raccolta dati}

Come anticipato, per confrontare $\mathrm{i}$ due gruppi in termini di autopercezioni nei confronti della scrittura e conseguente performance scolastica è stato somministrato il Questionario su quanto ti piace scrivere ed è stato raccolto un corpus di produzioni scritte.

I dati sono stati raccolti nel corso del secondo quadrimestre (febbraio-maggio) degli anni scolastici $2018 \backslash 2019$ e $2019 \backslash 2020$ : per prima cosa gli studenti hanno scritto il testo, successivamente hanno compilato il questionario. La procedura di rilevazione prevedeva che gli alunni di entrambi i gruppi scrivessero un testo narrativo a partire da un video stimolo, in questo caso un breve spezzone tratto dalla raccolta di comiche $W$ orld of Comedy (1962) di Harold Lloyd. Dopo due visioni del video, gli alunni hanno scritto il testo rispettando la medesima consegna: "Racconta la storia del video a qualcuno che non l'ha visto". Per l'intera somministrazione non sono stati previsti tempi precisi, anche se si è notato che le classi non hanno necessitato di più di due ore e mezza circa.

\subsection{Analisi}

I testi narrativi raccolti sono stati trascritti digitalmente, anonimizzati tramite codici alfanumerici e mescolati tra di loro, in modo tale da poter eseguire l'analisi senza sapere a chi, tra gruppo sperimentale e gruppo di controllo, appartenessero i vari elaborati, evitando così qualsiasi tipo di influenza (Lucisano, Salerni, 2002). Per valutare la qualità delle produzioni scritte si è fatto uso di quattro scale olistiche.

Le prime tre sono il risultato di traduzione e adattamento di tre delle quattro scale per la valutazione dell'adeguatezza funzionale di una produzione scritta proposte da Kuiken e Vedder (2016). Queste sono relative a tre aspetti: contenuto (completezza del testo in termini di informazioni fornite), comprensibilità (chiarezza del testo in termini di sforzi compiuti dal lettore per comprendere le idee espresse nel brano) e coerenza e coesione (buona struttura del testo in termini di connettivi utilizzati, legami logici tra le diverse parti del brano e riferimenti ai diversi contenuti e $\backslash$ o personaggi). Ogni scala è costituita da sei descrittori.

L'ultima scala è formata dai descrittori proposti dal Quadro Comune Europeo di riferimento per le lingue (QCER) (Consiglio d'Europa, 2002) per la categoria coerenza e coesione di una produzione linguistica. La scala si suddivide in otto descrittori: i primi tre sono relativi al livello elementare (A) del QCER; il quarto, quinto e sesto al livello intermedio (B); il sesto ed il settimo al livello avanzato (C). La differenza con la precedente scala, sempre relativa a coerenza e coesione, è che in questo secondo caso il focus dei descrittori è sui connettivi e loro funzione di collegamento logico all'interno di un testo. 
(C) Italiano LinguaDue 2. 2021. L. Lazzaretti, Tradurione, adattamento e validazione di un questionario su motivazione e disposizione verso la scrittura e suo impiego nello studio dell'autopercezione di classi intere e studenti multilingui

Per distinguere le due scale, quella tratta dai lavori di Kuiken e Vedder è stata denominata Coerenza e coesione 1, quella tratta dal QCER è stata denominata Coerenza e coesione 2. I quattro strumenti sono riportati in Appendice $\mathrm{B}^{7}$.

Per ciò che riguarda i questionari, per ogni item è stata misurata la posizione di ogni crocetta sulla rispettiva linea di risposta, individuando così un dato numerico per ogni quesito. Da tali cifre sono stati ricavati i valori medi, sia del singolo che per gruppi e sottogruppi, relativi ad ogni sottoscala dello strumento.

Per confrontare i due gruppi in termini sia linguistici che di autopercezione si è fatto uso del Welch t-test per campioni indipendenti, una volta appurata la normalità delle distribuzioni ${ }^{8}$. Nella sezione dedicata ai risultati, sia per gruppi interi che per sottogruppi, si riporteranno:

a) statistiche descrittive: media (M) e deviazione standard (DS);

b) il valore $p$ (due code) risultante dal Welch t-test e il relativo intervallo di confidenza al $95 \%(95 \% \mathrm{CI})$, ossia l'intervallo di valori entro cui dovrebbe cadere la differenza tra le medie nel 95\% delle future osservazioni con ulteriori campionamenti casuali di popolazione. La soglia per raggiungere la significatività statistica è posta a $p<0.05$;

c) l'ampiezza dell'effetto (effect size) ottenuto dall'intervento sperimentale tramite l'indice d di Cohen, che si ottiene dalla differenza tra media del gruppo sperimentale e media del gruppo di controllo divisa per la deviazione standard del gruppo di controllo;

d) il coefficiente di variazione (CV), che si calcola dividendo la deviazione standard di un determinato gruppo per la sua media. Tale misura serve per valutare l'omogeneità dei risultati del gruppo stesso attorno al suo valore medio: più il valore del coefficiente sarà basso, più i risultati dei singoli membri sanno omogenei attorno alla media.

\subsection{Risultati}

A seguire si riporteranno i risultati ottenuti dalla presente ricerca. Si inizierà con i dati linguistici, per poi proseguire con quelli di autopercezione.

\subsubsection{Risultati linguistici}

La Tabella 3 mostra i risultati del confronto tra gruppi e sottogruppi relativamente alle quattro scale olistiche.

\footnotetext{
7 Per assicurarsi dell'oggettività delle valutazioni di tipo olistico, un campione di 41 testi tratti dal corpus (circa il 20\% del totale) è stato oggetto di due tipi di analisi di affidabilità interna: inter-rater reliability (confronto tra le valutazioni di due diversi valutatori) e intra-rater reliability (confronto tra due valutazioni dello stesso individuo in due momenti differenti). Riferendosi a Koo e Li (2015), la valutazione è avvenuta mediante Intraclass correlation coefficient (ICC) nella variante two-way mixed effects model, single rating. L'ICC medio risultante dall' inter-rater reliability è 0.89 , quello risultante dall'intra-rater reliability è 0.91 .

${ }^{8}$ Secondo il parere di Fagerland e Sandvik (2009) è opportuno utilizzare il test di Welch in distribuzioni dalle 25 alle 100 unità che siano sostanzialmente normali o moderatamente asimmetriche, come nel nostro caso.
} 
(C) Italiano LinguaDue 2. 2021. L. Lazzaretti, Tradurione, adattamento e validazione di un questionario su motivazione e disposizione verso la scrittura e suo impiego nello studio dell'autopercezione di classi intere e studenti multilingui

Tabella 3. Risultati scale olistiche

\begin{tabular}{|l|c|c|c|c|c|c|c|}
\hline & $\begin{array}{c}\text { M (DS) } \\
\text { con }\end{array}$ & $\begin{array}{c}\text { CV } \\
\text { con }\end{array}$ & $\begin{array}{c}\text { M (DS) } \\
\text { exp }\end{array}$ & $\begin{array}{c}\text { CV } \\
\text { exp }\end{array}$ & 95\% CI & $\begin{array}{c}\text { d di } \\
\text { Cohen }\end{array}$ & $p$ \\
\hline \multicolumn{8}{|c|}{ Contenuto } \\
\hline Classe intera & $3.63(0.86)$ & 0.24 & $4.46(1.09)$ & 0.24 & $0.58,1.09$ & 0.97 & $* * * *$ \\
\hline Multilingui & $3.38(0.94)$ & 0.28 & $4.24(1.09)$ & 0.26 & $0.42,1.30$ & 0.92 & $* * * *$ \\
\hline \multicolumn{8}{|c|}{ Comprensibilità } \\
\hline Classe intera & $3.62(0.90)$ & 0.25 & $4.58(1.07)$ & 0.23 & $0.71,1.22$ & 1.08 & $* * * *$ \\
\hline Multilingui & $3.26(0.85)$ & 0.26 & $4.11(1.09)$ & 0.27 & $0.43,1.28$ & 1.01 & $* * * *$ \\
\hline \multicolumn{8}{|c|}{ Coerenza-coesione 1 } \\
\hline Classe intera & $3.63(0.67)$ & 0.19 & $4.34(0.83)$ & 0.19 & $0.52,0.92$ & 1.07 & $* * * *$ \\
\hline Multilingui & $3.23(0.71)$ & 0.22 & $4.04(0.85)$ & 0.21 & $0.48,1.15$ & 1.15 & $* * * *$ \\
\hline \multicolumn{8}{|c|}{ Coerenza-coesione 2 } \\
\hline Classe intera & $5.13(1.01)$ & 0.20 & $6.00(1.18)$ & 0.20 & $0.58,1.15$ & 0.86 & $* * * *$ \\
\hline Multilingui & $4.59(1.04)$ & 0.23 & $5.47(1.22)$ & 0.22 & $0.39,1.37$ & 0.84 & $* * * *$ \\
\hline
\end{tabular}

Nota. $* \mathrm{p}<0.05 ; \quad * * \mathrm{p}<0.01 ; \quad * * * \mathrm{p}<0.005 ; * * * * \mathrm{p}<0.001 ;$ ns $=$ non significativo

Osservando la Tabella 3 notiamo medie tutte a favore del gruppo sperimentale, sia intero che multilingui, e differenze tutte statisticamente significative. I risultati piuttosto marcati sono confermati anche dagli effect size, tutti alti (contenuto e coerenza-coesione 2) o molto alti (comprensibilità e coerenza-coesione 1). Rispetto all'omogeneità interna, $\mathrm{i}$ valori del coefficiente di variazione sono tutti abbastanza bassi e simili tra i gruppi, segno di compattezza dei risultati raggiunti dai diversi componenti di ogni raggruppamento. Sembrerebbe dunque che gli studenti che hanno seguito per tre o più anni i percorsi del Progetto scrivano testi mediamente più completi in termini di informazioni, più chiari $\mathrm{e}$ comprensibili nei loro legami logici e causali e meglio strutturati dal punto di vista di coerenza e coesione. Oltre a ciò, è ancor più positivo che tali risultati si verifichino anche nel sottogruppo dei multilingui, dando così l'idea che percorsi ed esperienze linguistiche inclusivi, collaborativi e di qualità possano contribuire a diminuire le difficoltà dovute a background linguistici e culturali differenti. Osservando i risultati si nota tuttavia che, sia per il gruppo sperimentale che per quello di controllo, le medie dei multilingui sono sempre inferiori di quelle del gruppo intero, anche se non di molto. Questo risultato non stupisce: pur essendo i risultati dei multilingui sperimentali migliori del rispettivo controllo, è naturale che, in entrambi i contesti, si manifesti qualche difficoltà in più nel sottogruppo che, presumibilmente, dovrebbe avere maggiori incertezze con la lingua.

\subsubsection{Risultati di autopercezione}

La Tabella 4 mostra i risultati del confronto tra gruppi e sottogruppi relativamente al Questionario su quanto ti piace scrivere. 
(C) Italiano LinguaDue 2. 2021. L. Lazzaretti, Tradurione, adattamento e validazione di un questionario su motivazione e disposizione verso la scrittura e suo impiego nello studio dell'autopercezione di classi intere e studenti multilingui

Tabella 4. Risultati questionario

\begin{tabular}{|l|l|l|l|l|l|l|l|l|}
\hline & M (DS) con & CV con & M (DS) exp & CV exp & 95\% CI & $\begin{array}{c}\text { d di } \\
\text { Cohen }\end{array}$ & $p$ & \\
\hline \multicolumn{7}{|c|}{ Fiducia } \\
\hline Classe intera & $169.09(53.40)$ & 0.32 & $154.57(47.30)$ & 0.31 & $-27.85,-1.18$ & -0.27 & $*$ & \\
\hline Multilingui & $159.95(53.55)$ & 0.33 & $154.49(46.88)$ & 0.30 & $-27.49,16.57$ & -0.10 & ns & \\
\hline \multicolumn{7}{|c|}{ Persistenza } \\
\hline Classe intera & $239.36(66.33)$ & 0.28 & $238.28(59.03)$ & 0.25 & $-17.67,15.52$ & -0.02 & ns & \\
\hline Multilingui & $241.08(68.94)$ & 0.29 & $254.84(61.88)$ & 0.24 & $-14.88,42.41$ & 0.20 & ns & \\
\hline \multicolumn{7}{|c|}{ Passione } \\
\hline Classe intera & $156.66(89.15)$ & 0.60 & $162.44(82.95)$ & 0.51 & $-16.94,28.50$ & 0.06 & ns & \\
\hline Multilingui & $161.13(85.30)$ & 0.53 & $183.93(80.81)$ & 0.44 & $-13.45,59.06$ & 0.27 & ns & \\
\hline
\end{tabular}

Nota. $*_{\mathrm{p}}<0.05 ; \quad * * \mathrm{p}<0.01 ; * * * \mathrm{p}<0.005 ;{ }^{* * * *} \mathrm{p}<0.001 ; \mathrm{ns}=$ non significativo

Passando ai risultati di autopercezione, notiamo una situazione molto più simile tra $\mathrm{i}$ due gruppi, con medie molto più vicine e quasi nessuna significatività statistica. Iniziando dalla fiducia, le medie sono a favore del gruppo di controllo in entrambi i casi, con significatività statistica per gruppo intero. Il $\mathbf{d}$ di Cohen è basso nel primo caso $(-0.27) \mathrm{e}$ molto basso nel secondo (-0.10). Passando alla persistenza, le medie sono pressoché identiche a gruppo intero, mentre vi è una media maggiore per il gruppo sperimentale per ciò che riguarda i multilingui. L'ampiezza dell'effetto è molto bassa nel primo caso $(-0.02)$ e bassa nel secondo (0.20). Infine, osservando i dati della sottoscala sulla passione, le medie sono maggiori per il gruppo sperimentale in entrambi i raggruppamenti, anche se non in modo estremamente marcato, come mostrato dall'assenza di significatività statistiche e dal $\mathbf{d}$ di Cohen molto basso (0.06) o basso (0.27). I valori del coefficiente di variazione sono maggiori rispetto a quelli emersi dai dati linguistici, segno di minore omogeneità di opinioni nel frangente dell'autopercezione. Nel caso di fiducia e persistenza i coefficienti dei due gruppi sono vicini tra loro e non eccessivamente alti, mentre nel caso della passione i valori sono abbastanza alti in entrambi i casi, ma sempre inferiori per il gruppo sperimentale.

\section{DISCUSSIONE E CONCLUSIONI}

Abbiamo iniziato questo discorso discutendo dei fattori emotivi e motivazionali legati alla scrittura e dell'importanza di una loro rilevazione a fini diagnostici durante il percorso scolastico degli studenti. Da diverso tempo, infatti, è noto alla ricerca che scrivere non è solo un processo cognitivo di trasmissione su carta dei propri pensieri, ma un atto sia mentale che sociale accompagnato e profondamente influenzato anche da aspetti motivazionali.

Proprio a tale scopo è stato prodotto il Writing dispositions scale for elementary and middle school students (Piazza, Siebert, 2008), questionario atto a ricavare informazioni da studenti di primaria e secondaria di primo grado circa la loro disposizione verso la scrittura, 

studenti multilingui

costrutto emergente da un intreccio di fattori legati a motivazione, interesse, autoregolazione e senso di autoefficacia. La prima parte del presente contributo riguarda proprio il processo di traduzione, adattamento e validazione di tale questionario, allo scopo di ricavarne una versione italiana fruibile in contesti sia didattici che di ricerca. Dal processo di traduzione letterale, adeguamento e perfezionamento di alcuni item, giudizio sulla comprensibilità dello strumento (fase pre-pilota) e valutazione della sua consistenza (fase pilota) è scaturito un questionario semplice, breve e di facile utilizzo, piuttosto chiaro e comprensibile e dalla complessiva buona "tenuta". Il fatto che, poi, i valori di affidabilità interna delle diverse sottoscale riprendano quelli emersi dallo strumento originario" ${ }^{9}$ i fa presupporre che il processo di costruzione e validazione sia stato svolto correttamente e portato a termine con discreto successo. Pur avendo giudicato i risultati di questo primo sondaggio tutti pienamente adeguati per i nostri scopi, il fatto che la sottoscala sulla persistenza raggiunga valori di poco sufficienti (Omega: $0.61,95 \%$ CI: 0.52-0.71) renderà necessario svolgere ulteriori rilevazioni di affidabilità dello strumento, contemplando nuovi campioni ed effettuando ulteriori modifiche e adattamenti.

Lo strumento costruito, denominato Questionario su quanto ti piace scrivere, è stato poi utilizzato, affiancato a scale di valutazione olistica, per una ricerca intenzionata ad indagare gli effetti, su abilità di scrittura e questioni motivazionali, che il progetto di educazione linguistica Osservare l'interlingua ha apportato su studenti di quinta primaria e terza secondaria di primo grado. Il Progetto, come anticipato, oltre a perseguire obiettivi linguistici si interessa anche a questioni trasversali all'apprendimento, come il sostegno degli alunni dal punto di vista affettivo e motivazionale e la volontà di integrare e coinvolgere ogni membro del gruppo nel lavoro comune, in particolare gli studenti con background linguistici e culturali differenti, al fine di ridurre le disuguaglianze e creare un ambiente positivo e accogliente. Lo studio serviva appunto per chiarire quanti e quali benefici ha apportato il Progetto sugli studenti che vi hanno preso parte, e soprattutto per comprendere se tali benefici si riflettono egualmente sia sul gruppo intero che sui soli multilingui, in altre parole se gli anni di Progetto sono serviti ad assottigliare le differenze tra i monolingui italofoni e coloro che presentano qualche difficoltà in più dal punto di vista linguistico.

Partendo dai dati linguistici, i risultati emersi sono estremamente positivi e tutti a favore del gruppo sperimentale, sia intero che per soli multilingui. Questo gruppo, in media, ottiene circa un punto in più rispetto a quello di controllo per ognuna delle quattro scale olistiche, raggiungendo la sufficienza in ogni caso. Ciò, avvalorato anche dagli effect size piuttosto alti e dai coefficienti di variazione bassi e simili tra loro, ci comunica che i testi narrativi degli studenti che hanno seguito per alcuni anni il Progetto sono meglio strutturati nelle componenti atte a garantire comprensibilità (tempi verbali adeguati, riferimento non ambiguo alle diverse entità, pochi salti logici e ripetizioni), più chiari e completi nei fatti narrati e più ricchi di connettivi e strutture utili a creare legami logici e causali tra le parti di brano. Inoltre, come già osservato in precedenza, i risultati ottenuti dai multilingui, in entrambi i gruppi, sono lievemente inferiori rispetto a quelli raggiunti a gruppo intero - come già scritto in sede di interpretazione dei risultati, è naturale aspettarsi che sia così, ma il solo fatto che anche il sottogruppo sperimentale ottenga risultati superiori al controllo, statisticamente significativi e piuttosto compatti, ci porta a credere che i presupposti e le attività del Progetto siano effettivamente un buon punto di partenza per ridurre le disuguaglianze e accrescere conoscenze e competenze di chi ha più difficoltà.

${ }^{9}$ La Persistenza è la sottoscala con i valori più bassi tra tutte; Fiducia e Passione raggiungono valori più alti, con la seconda scala più consistente della prima. 
Tali risultati sono certamente positivi e incoraggianti, ma chiaramente c'è ancora del lavoro da fare dal punto di vista linguistico: l'apprendimento non termina con i percorsi del Progetto e il processo di maturazione e miglioramento è ancora lungo.

Passando ora ai dati di autopercerione emersi dalla somministrazione del Questionario su quanto ti piace scrivere, i risultati sono decisamente meno marcati di quelli di natura linguistica, con ampiezza d'effetto molto bassa e quasi nessuna significatività statistica. L'unico risultato statisticamente rilevante è a favore del gruppo di controllo: a classi intere, questo gruppo riferisce di provare maggior fiducia nelle proprie capacità di scrittura rispetto al gruppo sperimentale. Per ciò che riguarda i restanti frangenti, le differenze sono tutte più lievi. Questi risultati, soprattutto fiducia e persistenza, sembrerebbero entrare in conflitto con gli esiti linguistici estremamente positivi raggiunti dagli studenti sperimentali; in altre parole, vi sarebbero le ragioni concrete, per questo gruppo, per riferire una migliore autopercezione rispetto al controllo, eppure questo, in linea di massima, non è accaduto. A tal proposito abbiamo tentato di formulare alcune interpretazioni per spiegare il fenomeno.

In primo luogo, ipotizziamo che gli anni di Progetto possano aver reso gli alunni sperimentali più critici e più capaci di mettere in discussione il proprio lavoro. L'approccio riflessivo e critico sulla lingua e i suoi usi comunicativi, i frequenti lavori di gruppo, i dibattiti e le discussioni potrebbero aver fatto ampliare le competenze metacognitive degli studenti sperimentali, ossia questi potrebbero essere divenuti in grado di leggere un compito di scrittura in termini di maggior complessità, riuscendo a considerare, nella sua riuscita, l'intervento di una più ampia quantità di variabili. Visti in questi termini, i risultati assumono una veste maggiormente positiva: anziché far leva sull'assoluta fiducia in sé e sull'idea di saper padroneggiare con abilità processi e strategie, il Progetto potrebbe aver inciso maggiormente sul senso critico e aver reso gli studenti cauti nell'autovalutarsi troppo positivamente in quanto scrittori. In tal senso, questi allievi sentirebbero che il loro rapporto con la scrittura è sempre in divenire e potrebbero dunque non reputarsi mai del tutto contenti e soddisfatti dei loro prodotti.

Si potrebbe anche ipotizzare, al contrario, che i presupposti e la metodologia del Progetto siano riusciti ad attecchire maggiormente dal punto di vista linguistico che non da quello motivazionale, per il quale forse sarebbe stato necessario ulteriore tempo. A tal proposito, vale la pena specificare che le attività del Progetto si sono svolte non in modo continuativo durante gli anni scolastici, ma circa per 2-3 ore settimanali, solitamente distribuite nella seconda parte dell'anno (gennaio \febbraio - maggio). Gli insegnanti più esperti e legati al Progetto da un maggior numero di anni hanno affermato che l'impianto metodologico della sperimentazione è diventato parte della loro quotidianità e si è ampliato anche ad altri momenti e discipline, e questo potrebbe aver portato ad un'influenza maggiore sul modo di lavorare degli studenti e sui loro atteggiamenti, tuttavia i momenti esclusivamente dedicati al Progetto non sono stati mai tantissimi nel corso degli anni scolastici. Inoltre, non tutte le classi sperimentali hanno preso parte alla sperimentazione per lo stesso numero di anni, il che potrebbe aver diversificato in parte le percezioni e l'esperienza dei singoli studenti.

Dal discorso sull'autopercezione emerge dunque la necessità di effettuare ulteriori indagini e rilevazioni, per comprendere quale delle ipotesi avanzate sia più vicina all'effettiva realtà. Tuttavia, nonostante non siano emersi risultati del tutto certi sul frangente motivazionale, occorre menzionare alcuni aspetti che sono abbastanza positivi per i presupposti del Progetto. Se è vero, infatti, che la maggior parte dei risultati scaturiti dal questionario non è particolarmente marcata - addirittura per la fiducia sono migliori 
(C) Italiano LinguaDue 2. 2021. L. Lazzaretti, Tradurione, adattamento e validazione di un questionario su motivazione e disposizione verso la scrittura e suo impiego nello studio dell'autopercezione di classi intere e studenti multilingui

gli esiti del controllo, con significatività a gruppo intero - è altrettanto vero che sono emersi anche alcuni aspetti positivi, seppur lievi, soprattutto a carico dei multilingui. Questi ultimi, infatti, riferiscono sia maggior persistenza che maggior passione rispetto al controllo, in maniera non estremamente ampia ma certamente in misura maggiore rispetto al gruppo intero, che eguaglia il controllo in termini di persistenza e lo supera di poco in termini di passione. Inoltre, i multilingui sperimentali eguagliano il rispettivo gruppo intero in termini di fiducia, ma esprimono più persistenza e più passione di quest'ultimo, stavolta con differenze abbastanza evidenti. Nel gruppo di controllo, invece, sono presenti divari inferiori tra i due raggruppamenti. Si potrebbe dire, insomma, che, seppur in modo non marcato, il Progetto abbia sortito alcuni effetti dal punto di vista motivazionale, in modo particolare sui multilingui. Questo risultato suggerisce che il presupposto di inclusione caro al Progetto è stato rispettato: gli esiti positivi si manifestano anche (in questo caso specifico di più) negli alunni ritenuti più in difficoltà, mostrando che le attività e i percorsi proposti sono utili a tutti, senza grosse distinzioni su abilità, lingua e cultura. Da questi risultati si potrebbe addirittura ipotizzare che gli effetti sull'autopercezione siano più evidenti sugli alunni multilingui, che forse necessitavano maggiormente di acquisire mezzi e conoscenze utili a mantenere uno sforzo più prolungato nella scrittura (persistenza) e per svolgere questo compito con maggior piacevolezza (passione). Nuovamente, però, la questione necessita di ulteriori approfondimenti per essere confermata.

\section{RIFERIMENTI BIBLIOGRAFICI}

Alderson J. C., Clapham C., Wall D. (1995), Language test construction and evaluation, Cambridge University Press, Cambridge.

Bailey K. D. (1995 [1978], Metodi della ricerca sociale II. L’inchiesta (M. Rossi, cur), il Mulino, Bologna.

Bandura A. (1986), Social foundations of thought and action: A social-cognitive theory, Prentice Hall, Englewood Cliffs, NJ.

Bazerman C. (2011), "Writing, cognition and affect from the perspectives of sociocultural and historical studies of writing", in Berninger V. W. (ed.), Past, present and future contributions of cognitive writing research to cognitive psychology, Taylor and Francis, New York, pp. 89-104:

https://bazerman.education.ucsb.edu/sites/default/files/docs/Bazerman2011Ch ptrWritCogAffect.pdf.

Bazerman C. (2016), "What do sociocultural studies of writing tell us about learning to write?", in MacArthur C. A., Graham S., Fitzgerald J. (eds.), Handbook of writing research 2 ed., Guilford, New York, pp. 11-23.

Benvenuto G. (2015), Stili e metodi della ricerca educativa, Carocci, Roma.

Black P., William D. (2009), "Developing the theory of formative assessment", in Journal of Personnel Evaluation in Education, 21, pp. 5-31:

https://link.springer.com/content/pdf/10.1007/s11092-008-9068-5.pdf.

Borghetti C., Ferrari S., Lazzaretti L., Pallotti G., Zanoni G. (2019), "Le voci dei personaggi: Insegnare l'uso del discorso riportato nella scuola primaria", in Italiano a scuola, 1, pp. 1-24: https://italianoascuola.unibo.it/article/view/9946/9847. 
(C) Italiano LinguaDue 2. 2021. L. Lazzaretti, Tradurione, adattamento e validazione di un questionario su motivazione e disposizione verso la scrittura e suo impiego nello studio dell'autopercezione di classi intere e studenti multilingui

Boscolo P. (a cura di) (1990), Insegnare i processi della scrittura nella scuola elementare, La Nuova Italia, Firenze.

Boscolo P. (2002), La scrittura nella scuola dell'obbligo, Laterza, Roma.

Boscolo P. (2012), La fatica e il piacere di imparare. Psicologia della motivarione scolastica, UTET, Torino-Novara.

Boscolo P., Hidi S. (2007), "The multiple meanings of motivation to write", in Boscolo P., Hidi S. (eds.), Writing and Motivation, Elsevier, Oxford, pp. 1-14.

Bruning R., Kauffman D. F. (2016), "Self-efficacy beliefs and motivation in writing development", in MacArthur C. A., Graham S., Fitzgerald J. (eds.), Handbook of writing research 2 ed., Guilford, New York, pp. 160-173.

Caselli M. (2007), Indagare col questionario. Introdurione alla ricerca sociale di tipo standard, Vita e Pensiero, Milano.

Chyung S. Y., Swanson I., Roberts K., Hankinson A. (2018), "Evidence-based survey design: The use of continuous rating scales in surveys", in Performance Improvement, 57,5 , pp. 38-48.

Cicchetti D. V., Sparrow S. S. (1990), "Assessment of adaptive behavior in young children", in Johnson J. J., Goldman J. (eds.), Developmental assessment in clinical child psychology: A handbook, Pergamon Press, New York, pp. 173-196.

Consiglio d'Europa. (2002), Quadro Comune Europeo di Riferimento per le lingue: apprendimento, insegnamento, valutazione, RCS Scuola, La Nuova Italia-Oxford, Milano.

Cook V. J. (1999), "Going beyond the native speaker in language teaching”, in TESOL Quarterly, 33, 2, pp. 185-209.

Cortina J. M. (1993), "What is Coefficient Alpha? An examination of theory and applications", in Journal of Applied Psychology, 78, 1, pp. 98-104.

https://www.psycholosphere.com/what $\% 20$ is $\% 20$ coefficient $\% 20$ alpha $\% 20$ by $\% 2$ 0Cortina.pdf.

Davies A. (2004), “The native speaker in applied linguistics", in Davies A., Elder C. (eds.), The handbook of applied linguistics, Wiley Blackwell, Oxford, pp. 421-450: https://www.poileasaidh.celtscot.ed.ac.uk/daviesseminar.html.

Dunn T. J., Baguley T., Brunsden V. (2013). "From alpha to omega: A practical solution to the pervasive problem of internal consistency estimation", in British Journal of Psychology, 105, 3, pp. 399-412.

Dweck C. S. (2000), Teorie del sé. Intelligenæa, motivazione, personalità e sviluppo, Erickson, Trento.

Dweck C. S., Grant H. (2008), "Self-theories, goals, and meaning", in Shah J. Y., Garrdner W. L. (eds.), Handbook of motivation science, Guilford, New York, pp. 405-416.

Ellis R. (2003), Task-based language teaching and learning, Oxford University Press, Oxford.

Fagerland M. W., Sandvik L. (2009), "Performance of five two-sample location tests for skewed distributions with unequal variances", in Contemporary Clinical Trials, 30, pp. 490-496.

Favaro G. (2018), "Le lingue, le norme, le pratiche. Il contesto, i dati, i riferimenti della scuola multiculturale e plurilingue", in Italiano LinguaDue, 2, pp. 1-41: https://riviste.unimi.it/index.php/promoitals/article/view/11283/10659.

Ferrari S., Burzoni G. (2018), "Imparare a scrivere meglio. Un'indagine sugli effetti di una sperimentazione educativa sulle classi intere e sugli alunni plurilingui", in ItalianoLinguaDue, 2, pp. 308-327:

https:// riviste.unimi.it/index.php/promoitals/article/view/11312/10696.

Galbraith D. (2009), "Cognitive models of writing", in German as a foreign language, 2-3, pp. 7-22. 
(C) Italiano LinguaDue 2. 2021. L. Lazzaretti, Tradurione, adattamento e validazione di un questionario su motivazione e disposizione verso la scrittura e suo impiego nello studio dell'autopercezione di classi intere e studenti multilingui

Giscel. (1975), Dieci tesi per l'educazione linguistica democratica: https://giscel.it/dieci-tesi-perleducazione-linguistica-democratica/.

Graham S. (2019), "Changing how writing is taught", in Review of Research in Education, 43, pp. 277-303:

https://journals.sagepub.com/doi/pdf/10.3102/0091732X18821125.

Green S. B., Lissitz R. W., Mulaik S. A. (1977), "Limitations of coefficient alpha as an index of test unidimensionality", in Educational and Psychological Measurement, 37, pp. 827-838.

Hidi S., Baird W. (1986), "Interestingness. A neglected variable in discourse processing", in Cognitive Science, 10, pp. 179-194. https://onlinelibrary.wiley.com/doi/pdfdirect/10.1207/s15516709cog1002_3.

Hidi S., Boscolo P. (2006), "Motivation and writing", in MacArthur C. A., Graham S., Fitzgerald, J. (eds.), Handbook of writing research 1 ed., Guilford, New York, pp. 144157.

King A. (1993), "From Sage on the stage to guide on the side", in College Teaching, 41, 1, pp. 30-35:

https:// faculty.washington.edu/kate1/ewExternalFiles/SageOnTheStage.pdf.

Koo T. K., Li M. Y. (2015), “A guideline of selecting and reporting Intraclass Correlation Coefficients for Reliability Research", in Journal of Chiropratic Medicine, 15, 2, pp. 155 163.

Kuiken F., Vedder I. (2016), "Functional adequacy in L2 writing: Towards a new rating scale", in Language Testing, 34, 3, pp. 1-16.

Lazarsfeld P. F. (1944), "The controversy over detailed interviews. An offer for negotiation", in Public Opinion Quarterly, 8, pp. 38-60.

Lucisano P., Salerni A. (2002), Metodologia della ricerca in educazione e formazione, Carocci, Roma.

Lumbelli L. (2009), La comprensione come problema. Il punto di vista cognitivo, Laterza, BariRoma.

MacArthur C. A., Graham S. (2016), "Writing research from a cognitive perspective", in MacArthur C. A., Graham S., Fitzgerald J. (eds.), Handbook of writing research $2 \mathrm{ed}$, Guilford, New York, pp. 11-23.

McCarthy P., Schmeck R. R. (1988), 'Students' self-concepts and the quality of learning in public schools and universities", in Schmeck R. R. (ed.), Learning Strategies and learning styles, Plenum Press, New York, pp. 131-158.

McDonald R. P. (1970), "The theoretical foundations of principal factor analysis, canonical factor analysis, and alpha factor analysis", in British Journal of Mathematical and Statistical Psychology, 23, 1, pp. 1-21.

McDonald R. P. (1999), Test theory: A unified treatment, Erlbaum, Mahwah, NJ.

Montrul S., Polinsky M. (2011), "Why not heritage speakers?", in Linguistic Approaches to Bilingualism, 1, 1, pp. 58-62.

https://dash.harvard.edu/bitstream/handle/1/4726010/LAB_commentary_Mon trul_Polinsky $\% 20102410$.pdf; sequence $=1$.

Nunan D. (2004), Task-based language teaching, Cambridge University Press, Cambridge.

Pallotti G. (2017a), "Applying the interlanguage approach to language teaching", in International review of applied linguistics in language teaching, 55, 4, pp. 393-412.

Pallotti G. (2017b), "Osservare l'interlingua. Percorsi di educazione linguistica efficace per ridurre le diseguaglianze", in Vedovelli M. (a cura di), L'italiano dei nuovi italiani, Aracne, Roma, pp. 505-520: 
(C) Italiano LinguaDue 2. 2021. L. Lazzaretti, Tradurione, adattamento e validazione di un questionario su motivazione e disposizione verso la scrittura e suo impiego nello studio dell'autopercezione di classi intere e studenti multilingui

https://iris.unimore.it/retrieve/handle/11380/1153170/181478/16GiscelPallotti EfficaciaOssIL-1.pdf.

Pallotti G., Borghetti C. (2019), "The effects of an experimental approach to writing instruction on monolingual and multilingual pupils in Italian primary schools", in EuroAmerican Journal of Applied Linguistics and Languages, 6, 1, pp. 1-20: https://web.archive.org/web/20200211225700id_/http://www.ejournall.org/wp-content/uploads/Pallotti_Borghetti_6.1.pdf.

Pallotti G., Borghetti C. Rosi F. (2021), Insegnare a scrivere nella scuola primaria. Il progetto Osservare l'interlingua, Caissa Italia, Bologna: https://www.caissa.it/357-insegnare-a-scrivere-nella-scuola-primaria-il-progettoosservare-l-interlingua-9788867291021.html.

Pallotti G., Ferrari S. (2019), Osservare l'Interlingua. Un sillabo per un' educazione linguistica inclusiva ed efficace: https://interlingua.comune.re.it/?page_id=3493.

Pallotti G., Rosi F. (2017a), "Più competenze, meno disuguaglianze. Risultati di una sperimentazione di educazione linguistica inclusiva nella scuola secondaria di primo grado", in Vedovelli M. (ed.), L'italiano dei nuovi italiani, Aracne, Roma, pp. 193-209: https:/ /view.officeapps.live.com/op/view.aspx?src $=$ https $\% 3 \mathrm{~A} \% 2 \mathrm{~F} \% 2$ Firis.unim ore.it $\% 2$ Fretrieve $\% 2$ Fhandle $\% 2$ F11380\%2F1153176\%2F181488\%2F16PallottiRosi-Giscel\%2520Medie4.docx\&wdOrigin=BROWSELINK.

Pallotti G., Rosi F. (2017b), "Educazione linguistica inclusiva nella scuola primaria: percorsi di sperimentazione e analisi dell'efficacia", in Corrà L. (ed.), Educažione linguistica in classi multietniche, Aracne, Roma, pp. 117-142:

https:/ /view.officeapps.live.com/op/view.aspx?src $=$ https $\% 3 \mathrm{~A} \% 2 \mathrm{~F} \% 2$ Firis.unim ore.it $\% 2$ Fretrieve $\% 2$ Fhandle $\% 2$ F11380\%2F1153172\%2F181482\%2F15PallottiRosi\%2520atti\%2520Giscel_SLI-2.docx\&wdOrigin=BROWSELINK.

Phillips D. L., Clancy K. J. (1972), "Some Effects of 'Social Desirability' in Survey Studies", in American Journal of Sociology, 77, 5, pp. 921-940.

Piazza C. L., Siebert C. F. (2008), "Development and validation of a writing dispositions scale for elementary and middle school students", in The Journal of Educational Research, 101, 5, pp. 275-286.

Presser S., Zhao S. (1992), "Attributes of questions and interviewers as correlates of interviewing performance", in The Public Opinion Quarterly, 56, 2, pp. 236-240.

Prior P. (2006), "A sociocultural theory of writing", in MacArthur C., Graham S., Fitzgerlad J. (eds.), Handbook of Writing Research, Guilford, New York, pp. 54-66. https:/ / books.google.it/books?hl=it\&lr=\&id=VT7YCz2G-

$\mathrm{iQC} \& \mathrm{i}=$ fnd \&pg $=\mathrm{PA} 54 \& \mathrm{dq}=\mathrm{A}+$ sociocultural + theory $+\mathrm{of}+$ writing\&ots $=\mathrm{Rb} 8 \mathrm{OL}$ j8-fE\&sig=R9I_x-StaVENABKNKD4pljBlL-

c\&redir_esc $=\mathrm{y} \# \mathrm{v}=$ onepage $\& \mathrm{q}=\mathrm{A} \% 20$ sociocultural $\% 20$ theory $\% 20 \mathrm{of} \% 20 \mathrm{writing}$ $\& \mathrm{f}=$ false.

Renninger K. A., Hidi S., Knapp A. (a cura di) (1992), The role of interest in learning and development. Erlbaum, Hillsdale, NJ.

Sansone C., Thoman D. B. (2005), "Interest as the missing motivator in self-regulation", in European Psychologist, 10, 3, pp. 175-186.

https://www.researchgate.net/profile/DustinThoman/publication/232464951_I nterest_as_the_Missing_Motivator_in_SelfRegulation/links/02e7e5384cd5b3cd9 b000000/Interest-as-the-Missing-Motivator-in-Self-Regulation.pdf.

Santangelo T., Harris K. R., Graham S. (2016). "Self-regulation and writing. Meta-analysis of the self-regulation processes in Zimmerman and Risemberg's model", in 
(C) Italiano LinguaDue 2. 2021. L. Lazzaretti, Tradurione, adattamento e validazione di un questionario su motivazione e disposizione verso la scrittura e suo impiego nello studio dell'autopercezione di classi intere e studenti multilingui

MacArthur C. A., Graham S., Fitzgerald J. (eds.), Handbook of writing research 2 ed., Guilford, New York, pp. 174-193.

Selinker L. (1972), "Interlanguage", in International Review of Applied Linguistics, 10, 2, pp. 209-231.

Streiner D. L. (2003), "Starting at the beginning: An introduction to Coefficient Alpha and Internal Consistency", in Journal of Personality Assessment, 80, 1, pp. 99-103.

Torrance M., Galbraith D. (2006), "The processing demands of writing”, in MacArthur C., Graham S., Fitzgerlad J. (eds.), Handbook of Writing Research, Guilford, New York, pp. 67-82.

Van Rossum E. J., Schenk S. M. (1984), "The relationship between learning conception, study strategy and learning outcome", in British Journal of Educational Psychology, 54, pp. 73-83.

Vaske J. J., Beaman J., Sponarski C. C. (2017), "Rethinking internal consistency in Cronbach's Alpha", in Leisure Sciences, 39, 2, pp. 163-173.

Vygotskij L. S. (1992 [1934]), Pensiero e linguaggio (Trad. it di Mecacci L.), Laterza, BariRoma.

Vygotskij L. S. (2006 [1926]), Psicologia pedagogica: Manuale di psicologia applicata all'insegnamento e all'educazione (Trad. it di Veggetti M. S.), Erickson, Trento.

White M. J., Bruning R. (2005), "Implicit writing beliefs and their relation to writing quality", in Contemporary Educational Psychology, 30, pp. 166-189.

White R., Arndt V. (1991), Process writing, Longman, Harlow.

Zimmerman B. J. (2002), "Becoming a self-regulated learner: An overview", in Theory into Practice, 41, 2, pp. 64-70.

Zimmerman B. J., Kitsantas A. (1997), "Developmental phases in self-regulation: Shifting from process to outcome goals", in Journal of Educational Psychology, 89, pp. 29-36.

\section{APpendice A}

QUESTIONARIO SU QUANTO TI PIACE SCRIVERE

(tradotto e adattato da Piazza, Siebert, 2008)

1. I miei testi sono scritti molto bene. $(\mathrm{F})$

2. Mi diverto a scrivere. (PA)

3. Anche se ci vuole un po' di tempo, cerco di pensare a diversi modi di dire la stessa cosa, per capire qual è il migliore. (PE)

4. Mi piacerebbe scrivere di più a scuola. (PE)

5. Per me scrivere è facile. $(\mathrm{F})$

6. Scrivere è la mia attività scolastica preferita. (PA)

7. Se devo scrivere un testo lungo, ci metto tutto il mio impegno, anche se ci vuole più tempo. (PE)

8. Se posso scegliere cosa fare nel mio tempo libero, scelgo di scrivere. (PA)

9. In classe non vedo l'ora di scrivere. (PA)

10. Cerco di correggere i miei testi al meglio, anche se ci vuole un po' di tempo. (PE)

11. Non sono tanto bravo a scrivere. (F)

Legenda: $(\mathrm{F})=$ Fiducia, $(\mathrm{PE})=$ Persistenza,$(\mathrm{PA})=$ Passione 
(C) Italiano LinguaDue 2. 2021. L. Lazzaretti, Tradurione, adattamento e validazione di un questionario su motivazione e disposizione verso la scrittura e suo impiego nello studio dell'autopercezione di classi intere e studenti multilingui

\section{APPENDiCE B}

\section{SCALE IMPIEGATE PER LA VALUTAZIONE OLISTICA}

Scala contenuto (tratta da Kuiken, Vedder, 2016; 2018)

\begin{tabular}{|l|l|}
\hline 1 & Per niente adeguato: il numero di idee è del tutto insufficiente e non adeguato. \\
\hline 2 & Poco adeguato: il numero di idee è scarsamente adeguato. \\
\hline 3 & Parzialmente adeguato: il numero di idee è abbastanza adeguato. \\
\hline 4 & Adeguato: il numero di idee è adeguato. \\
\hline 5 & Molto adeguato: il numero di idee è molto adeguato. \\
\hline 6 & Assolutamente adeguato: il numero di idee è assolutamente adeguato. \\
\hline
\end{tabular}

Scala comprensibilità (tratta da Kuiken, Vedder, 2016; 2018)

$1 \quad$ Il testo non è affatto comprensibile. Le idee e lo scopo sono espressi in modo oscuro e il lettore, anche sforzandosi, non riesce a capire.

2 Il testo non si comprende facilmente, i suoi scopi non sono chiari e il lettore deve sforzarsi molto per capire le idee dell'autore. Il lettore deve cercare di indovinare la maggior parte delle idee e degli scopi del testo.

3 Il testo è abbastanza comprensibile, ma alcune frasi non si capiscono bene a una prima lettura. Un'ulteriore rilettura è utile per chiarire gli scopi del testo e le idee espresse, ma rimangono alcuni dubbi.

$4 \quad$ Il testo è comprensibile, solo certe frasi sono poco chiare ma si possono capire senza grandi sforzi con una rilettura.

5 Il testo è facile da comprendere e si legge agevolmente, non ci sono problemi di comprensibilità.

6 Il testo è molto facile da comprendere e si legge molto agevolmente, le idee e gli scopi sono espressi con chiarezza.

Scala Coerenza e coesione 1 (tratta da Kuiken, Vedder, 2016; 2018)

$1 \quad$ Il testo non è per nulla coerente: ci sono frequenti salti logici e argomenti non collegati. L'autore non usa riferimenti anaforici (pronomi, frasi con soggetto sottinteso chiaramente interpretabile). Il testo non è affatto coeso, i connettivi sono praticamente assenti e le idee non sono collegate tra loro.

2 Il testo è poco coerente. L'autore spesso non collega gli argomenti tra loro; se c'è coerenza, questa è espressa per lo più da ripetizioni. Pochi riferimenti anaforici e alcuni salti logici. Il testo è poco coeso: vengono usati pochi connettivi, che non collegano bene le idee.

3 Il testo è abbastanza coerente. Ci sono frequenti salti di argomento e/o ripetizioni. Più di due frasi di seguito esprimono esplicitamente lo stesso soggetto, anche quando questo sarebbe chiaro. Vengono usati alcuni riferimenti anaforici. Possono esservi alcune interruzioni della coerenza. Il testo è abbastanza coeso: vengono usati alcuni connettivi, ma sono per lo più delle congiunzioni. 
(C) Italiano LinguaDue 2. 2021. L. Lazzaretti, Tradurione, adattamento e validazione di un questionario su motivazione e disposizione verso la scrittura e suo impiego nello studio dell'autopercezione di classi intere e studenti multilingui

4 Il testo è coerente. I salti di argomento sono abbastanza rari, ma l'autore a volte riesce a essere coerente solo mediante ripetizioni non necessarie. Si trovano sufficienti riferimenti anaforici. Possono esservi interruzioni della coerenza. L'autore fa un buon uso dei connettivi, che a volte vanno oltre le semplici congiunzioni.

5 Il testo è molto coerente: quando si introduce un nuovo argomento, di solito ciò avviene mediante l'uso di connettivi o espressioni di collegamento esplicite. Le ripetizioni sono molto rare e si trovano numerosi riferimenti anaforici, con nessuna interruzione della coerenza. Il testo è molto coeso e le idee sono ben collegate tra loro mediante connettivi verbali o avverbiali.

6 L'autore produce un'ottima coerenza integrando le nuove idee nel testo con connettivi o espressioni di collegamento esplicite. I connettivi anaforici sono usati regolarmente, con qualche raro caso di argomenti non collegati e nessuna interruzione della coerenza. La struttura del testo è estremamente coesa, grazie a un abile uso dei connettivi (in particolare verbali, avverbiali e formule di collegamento), usati spesso per descrivere le relazioni tra le idee.

Scala Coerenæa e coesione 2 (tratta da QCER, Consiglio d'Europa, 2002)

\begin{tabular}{|l|l|}
\hline 1 & $\begin{array}{l}\grave{E} \text { in grado di collegare parole o gruppi di parole con connettivi molto elementari quali } e \text { o } \\
\text { allora. }\end{array}$ \\
\hline 2 & È in grado di collegare gruppi di parole con connettivi semplici quali e, ma, perché. \\
\hline 3 & $\begin{array}{l}\text { È in grado di collegare frasi semplici usando i connettivi più usuali per raccontare una storia } \\
\text { o descrivere qualcosa, realizzando un semplice elenco di punti. }\end{array}$ \\
\hline 4 & $\begin{array}{l}\text { È in grado di collegare una serie di elementi relativamente brevi e semplici in una sequenza } \\
\text { lineare per punti }\end{array}$ \\
\hline 5 & $\begin{array}{l}\text { E in grado di usare un numero limitato di elementi di coesione per collegare i propri } \\
\text { enunciati in un discorso chiaro e coerente. In un intervento lungo possono presentarsi dei } \\
\text { salti' logici. }\end{array}$ \\
\hline 6 & $\begin{array}{l}\text { È in grado di usare in modo efficace diversi connettivi per esplicitare i rapporti tra i } \\
\text { concetti. }\end{array}$ \\
\hline 7 & $\begin{array}{l}\grave{E} \text { in grado di realizzare un discorso chiaro, sciolto e ben strutturato, mostrando un uso } \\
\text { controllato degli schemi organizzativi, di connettivi ed espressioni coesive. }\end{array}$ \\
\hline 8 & $\begin{array}{l}\grave{E} \text { in grado di realizzare un discorso coerente e coeso usando in modo appropriato una } \\
\text { grande varietà di schemi organizzativi e un'ampia gamma di connettivi e di meccanismi } \\
\text { coesivi di altro tipo. }\end{array}$ \\
\hline
\end{tabular}

